\title{
Los Lamini (Cetartiodactyla: Camelidae) extintos del yacimiento de Pilauco (Norpatagonia chilena): aspectos taxonómicos y tafonómicos preliminares
}

\author{
The extinct Lamini (Cetartiodactyla: camelidae) from Pilauco site \\ (Chilean north Patagonia): preliminary taxonomic and taphonomic \\ features
}

\author{
Rafael Labarca Encina¹, Mario Pino¹, Omar Recabarren¹
}

RESUMEN

Se presenta la descripción y asignación taxonómica preliminar de los restos de Lamini recuperados en el sitio paleontológico de Pilauco (Pleistoceno tardío) ubicado en el sur de Chile. Sobre la base de variables métricas los materiales son provisoriamente incluidos en cf. Hemiauchenia paradoxa Gervais \& Ameghino 1880 a la espera de materiales más diagnósticos. Se discuten algunos procesos tafonómicos ocurridos al interior del yacimiento a partir del estudio de las marcas registradas en la superficie de los fósiles, las que son concordantes con el ambiente palustre con inundaciones esporádicas fluviales descrito para el sitio.

Palabras clave: Lamini, Pleistoceno tardío, Tafonomía, Norpatagonia, Chile.

\begin{abstract}
We present a preliminary description and taxonomic assignment of the Lamini fossil remains recovered from the paleontological site of Pilauco (late Pleistocene) in southern Chile. Based on metric variables the fossils are temporarily asigned to cf. Hemiauchenia paradoxa Gervais \& Ameghino 1880, waiting for new and more diagnostic remains. We present and discuss some taphonomic processes occurring within the site, starting from the study of the marks observed in the surface of the fossils, wich are consistent with the bog environment with occasional fluvial flooding described for the site.
\end{abstract}

Keywords: Lamini, late Pleistocene, taphonomy, northern Patagonia, Chile.

\section{Introducción}

La mastofauna terrestre del Pleistoceno Sudamericano $(2,7 \mathrm{Ma}-11.500$ años AP) se conforma a partir de dos grandes linajes con distintas historias evolutivas. Por un lado se encuentran aquellos grupos endémicos del subcontinente, los que evolucionan en un aislamiento relativo tras la separación de Sudamérica de África en el Cretácico superior y de la Antártida en el Oligoceno superior, hace unos 30 Ma (Pascual \& Ortíz-Jaureguízar, 2007). Dentro de este grupo se encuentran los Xenarthra, Marsupialia, Litopterna y parte de los Rodentia (Cione \& Tonni 1995; Woodburne et al., 2006). Este conjunto se complementa con formas alóctonas de origen holártico, las que comienzan a acceder de manera esporádica el subcontinente desde Norteamérica hace unos $9 \mathrm{Ma}$, y de manera más intensiva tras la aparición del istmo de Panamá, hace unos 2,7 Ma, evento conocido como Gran Intercambio Biótico Americano (GABI) (Cione \& Tonni, 1995; Marshall et al., 1984; Webb, 1991; Woodburne et al., 2006). Dentro de este ensamble foráneo se encuentran los Proboscidea, Cetartiodactyla y Perissodactyla, así como la mayoría de los representantes de los Canidae, Felidae y Sigmodontinae (Cione \& Tonni, 1995; Marshall et al., 1984; Webb, 1991; Woodburne et al., 2006).

\footnotetext{
${ }^{1}$ Laboratorio de Paleoecología, Instituto de Ciencias Ambientales y Evolutivas, Universidad Austral de Chile, Casilla 567, Valdivia, Chile. Email: r.labarca.e@gmail.com
} 

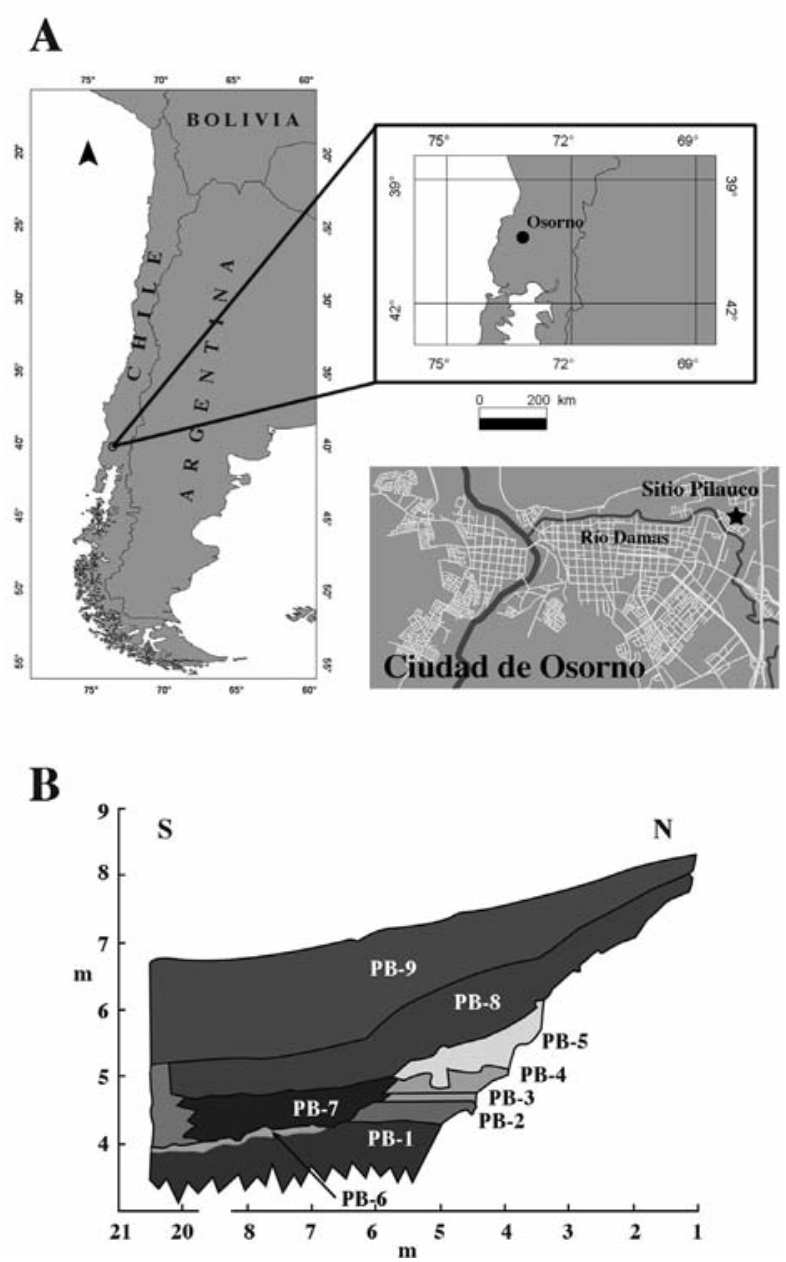

Fig. 1.-A. Ubicación del sitio Pilauco; B. Esquema estratigráfico del sitio Pilauco. PB1: Toba de lapilli. PB-2: Toba de lapilli con abundante matriz de ceniza volcánica. PB-3: Toba de lapilli con matriz de arena media. PB-4: Ceniza volcánica. PB-5: Arena gruesa terrígena, con guijarros angulosos dispersos. PB6: Grava compuesta por clastos de 1 a $15 \mathrm{~cm}$ de diámetro. PB7: Turba de matriz marrón muy oscuro (10YR 3/1) con clastos aislados (menos 15\%) de origen andino de hasta $7 \mathrm{~cm}$, muy mal seleccionados. De esta capa provienen los fósiles de Lamini descritos en el presente trabajo. PB-8: Turba de composición muy similar a PB-7, pero de color levemente más marrón $(2.5 \mathrm{Y}$ 4/2). PB-9: Turba de color negro (2.5 Y 2/0) (modificado de Recabarren et al., 2011).

Dentro del orden Cetartiodactyla se ubica la Tribu Lamini Webb, 1965 (Mackenna \& Bell, 1997), representada en Sudamérica por los géneros Lama Frisch, 1775, vicugna Gray, 1872, Eulamaops Ameghino, 1889, Palaeolama Gervais, 1867 y Hemiauchenia Gervais \& Ameghino, 1880 (Mackenna \& Bell, 1997; Scherer, 2009; Menegaz, 2000; Menegaz \& Ortíz-Jaureguizar, 1995). En la prehistoria de Sudamérica el género Lama se registra desde el Plioceno final (Ensenadense) al Pleistoceno final (Lujanense) de Argentina, en el Pleistoceno tardío de Uruguay, Brasil y Chile; en el Pleistoceno medio (Bonaerense) de Bolivia y actualmente se reconoce en el Holoceno del Sur de Ecuador, Perú, Bolivia, Chile y Uruguay (González et al., 2006; Scherer, 2009; Scherer et al., 2007; Ubilla et al., 2004; Mengaz \& Ortíz Jaureguizar, 1995; Menegaz, 2000; MacFadden \& Shockey, 1997). Los hallazgos prehistóricos en Sudamérica de Vicugna se registran posiblemente desde el Pleistoceno medio al Pleistoceno tardío de Argentina y Bolivia, y en el Pleistoceno tardío de Chile, Sur de Brasil y Uruguay (Cartajena et al., 2010; Tauber, 1999; Labarca \& Prieto, 2009; Weinstock et al., 2009; Menegaz, 2000; Scherer, 2009, Ubilla et al., 2004). Palaeolama se registra en el Ensenadense del sur de Perú, desde el Bonaerense al Lujanense de la región andina de Bolivia y en el Lujanense de Brasil, Chile, Perú, Ecuador, Venezuela, Perú y Uruguay (Marshall y Sempere, 1991; Marshall et al., 1984; MacFadden \& Shockey, 1997; Scherer 2005, 2009; Menegaz, 2000; Labarca \& Lopez, 2006; López et al., 2005). Finalmente, Hemiauchenia posee registros posiblemente desde el Plioceno tardío al Pleistoceno tardío de Argentina, y en el Pleistoceno tardío de Brasil, Uruguay y Bolivia (Scherer, 2009; Menegaz, 2000; Marshal et al., 1984).

Recientemente Scherer (2009) realizó una completa revisión a las formas sudamericanas de la Tribu, discutiendo los caracteres diagnósticos utilizados históricamente en la literatura. Sus resultados permitieron simplificar la taxonomía del clado, sinonimizando una gran cantidad de formas descritas hacia finales del siglo XIX y XX. Al mismo tiempo, propuso nuevos caracteres diagnósticos para los distintos géneros y especies, relacionados tanto con variables morfológicas como métricas. De esta manera, para Palaeolama reconoce en Sudamérica solo las especies $P$. weddelli Gervais, 1855, P. major Liais, 1872 y con reservas, P. hoffstetteri Guerin \& Faure, 1999; por su parte, dentro de Hemiauchenia solo es válida la especie H. paradoxa Gervais y Ameghino, 1880. En el género Lama incluye Lama guanicoe Muller, 1776, Lama castelnaudi Boule \& Thevenin, 1920, y Lama glama Linnaeus, 1775; mientras que para vicugna se consideran válidas solo Vicugna vicugna Molina, 1782, Vicugna provicugna Boule \& Thevenin, 1920 y Vicugna pacos Linnaeus, 1775 (Scherer, 2009). 


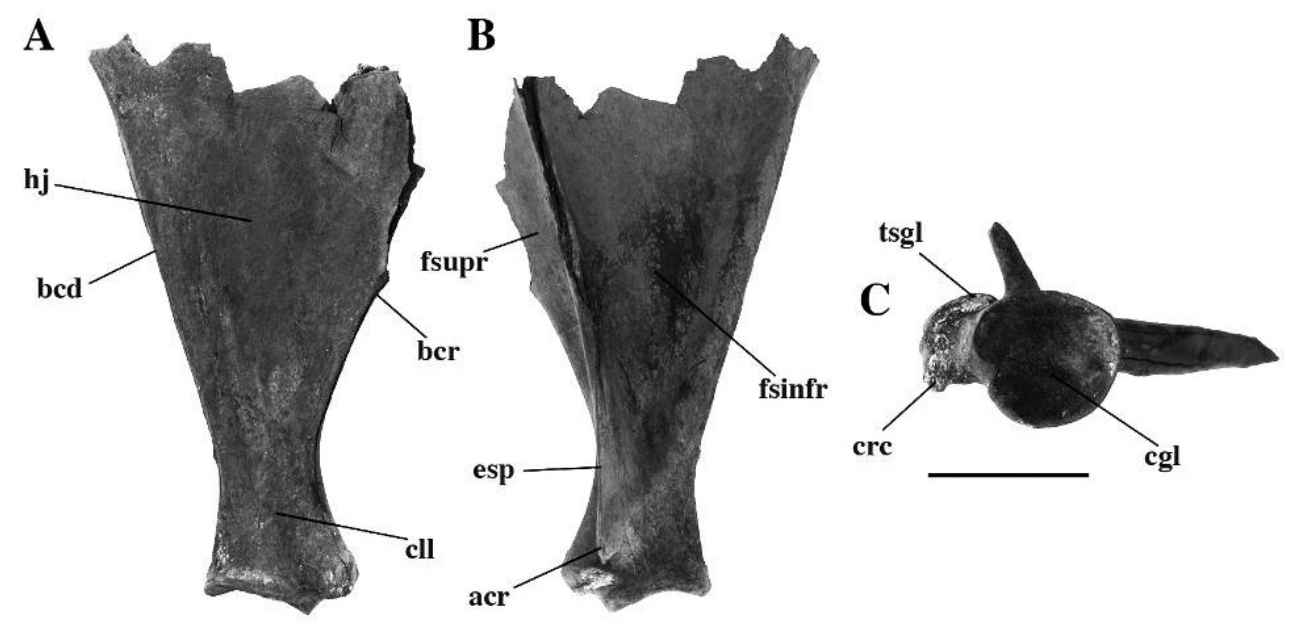

D

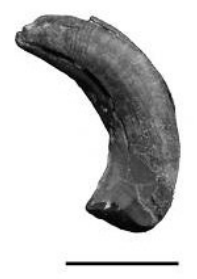

G

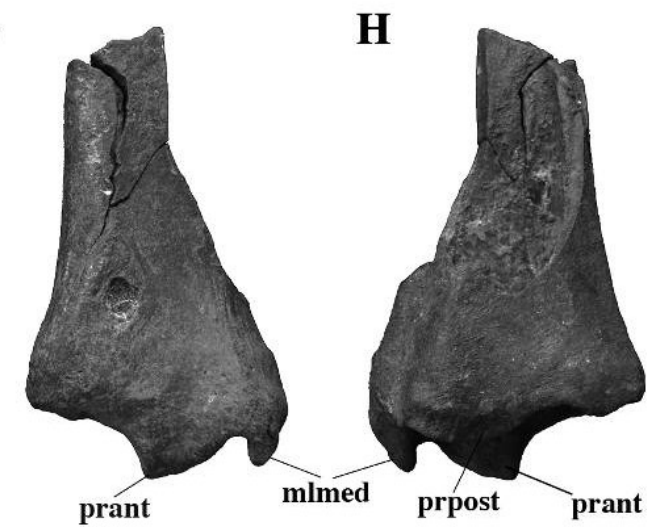

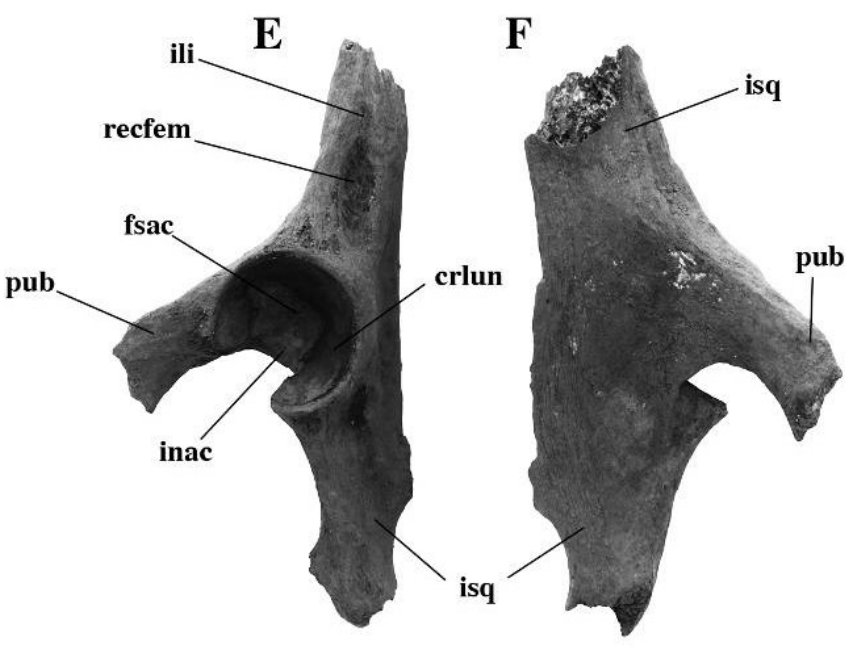

F

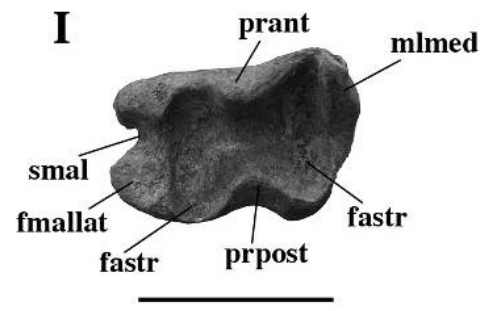

Fig. 2.-cf. Hemiauchenia paradoxa. Escápula izquierda (MHMOP/PI/602), vistas medial (A), lateral (B) y distal (C); incisivo/incisivo caniniforme (MHMOP/PI/540) (D); coxal derecho (MHMOP/PI/601), vista lateral (E) y medial (F); tibia derecha (MHMOP/PI/72), vistas anterior (G), posterior (H) y distal (I). hj, hoja; bcd, borde caudal; bcr, borde craneal, cll, cuello; fsupr, fosa supraespinosa; fsinf, fosa infraespinosa; esp, espina; acr, acromion; tsgl, tubérculo supraglenoideo; crc, coracoides; cgl, cavidad glenoidea; ili, ilion; isq, isquion; pub, pubis; recfem, incisura para el músculo recti femoris; crlun, carilla lunar; fsac, fosa acetabular; inac, incisura acetabular; pran, proceso anterior; prpost; proceso posterior; mlmed, maléolo medial; smal, surco maleolar; fmallat, faceta articular para el maléolo lateral; fastr, faceta astragalar; mlmed, maléolo medial. La escala mide $5 \mathrm{~cm}$, a excepción de $\mathrm{D}$, en donde mide $0,5 \mathrm{~cm}$. 
El registro de camélidos fósiles pleistocénicos en Chile es escaso (López et al., 2005), a excepción de tres áreas geográficamente acotadas: norte grande (circa $\left.26^{\circ} 30^{\prime} \mathrm{S}\right)$, norte semiárido (circa $32^{\circ} \mathrm{S}$ ) y el área sur patagónica (al sur de los $53^{\circ} \mathrm{S}$ ). En el primero de estos se ha mencionado la presencia de "Lama gracilis", en contextos de desagüe de antiguas lagunas altoandinas del Pleistoceno tardío (Cartajena et al., 2010). Este taxón, sin embargo, es sinónimo de Vicugna vicugna de acuerdo a Scherer (2009), por lo que los materiales, a nuestro juicio, deberían ser asignados a Vicugna provicugna. Para el norte semiárido chileno se ha descrito a Palaeolama sp. en depósitos fluviales del Pleistoceno tardío - Holoceno temprano (Núñez et al., 1983; Labarca \& López, 2006; López, 2007), mientras que para el sector austral se ha indicado Lama guanicoe, Vicugna vicugna y Lama sp. (taxón que presentaría un mayor tamaño que el guanaco actual, aunque podría corresponder a Lama guanicoe de gran talla) en distintos reparos rocosos asociados en algunos casos a herramientas de piedra durante la transición Pleistoceno - Holoceno (Mengoni - Goñalons, 1987; Latorre, 1998; Borrero et al., 1997; Weinstock et al., 2009; entre otros). Fuera de este registro existen referencias de elementos aislados y poco diagnósticos, inscritos en sentido amplio al Pleistoceno tardío (Casamiquela, 1999)

Este trabajo describe los restos óseos de los Lamini extintos del sitio paleontológico de Pilauco (Pleistoceno tardío), ubicado en el extremo norte del área patagónica chilena (Figura 1A). Se presenta una asignación taxonómica preliminar sobre la base de variables métricas y se discuten algunos procesos tafonómicos a partir de las marcas observadas en la superficie de los restos óseos.

\section{Marco geomorfológico y estratigráfico}

Según Recabarren et al. (2011) en el sitio de Pilauco es posible identificar dos unidades geomorfológicas, cada una con su propio conjunto de estratos. El límite norte del sitio está conformado por colinas cuyas capas fueron depositadas por actividad volcánica andina de composición ácido a intermedia, en la forma de una serie de flujos volcanoclásticos, agrupados bajo el nombre de "Secuencia piroclástica - epiclástica San Pablo" (Pérez et al., 2003). La edad de esta unidad es mayor que las de los depósitos del último glacial Llanquihue (Mercer, 1976). Seis estratos lenticulares, incluidos los sedi- mentos volcanoclásticos terrígenos, componen la base de las colinas en el sitio (PB-1 a PB-6, Figura 1B). Al sur de las colinas, depósitos de llanura de inundación originaron cuatro estratos. Sobre una discordancia de erosión labrada en la capa PB-1 (la base de la colina), se dispone una capa lenticular de grava con escasa matriz arenosa (PB-6). Por encima de esta se encuentran PB-7, -8 y -9 , las que forman estratos horizontales bien definidos. PB-7 y PB-8 están compuestos por turba con componentes menores de $15 \%$ de grava y arenas andinas. Inmediatamente $(50 \mathrm{~m})$ hacia el sur las dos capas se interdigitan con sedimentos compuestos por grava sin matriz fina interpretados como clásicos depósitos fluviales (Figura 1B). Un total de 16 fechados en hueso, madera y carbón sitúan la capa PB-7 entre el $13.220 \pm 60$ (AA101831) y $11.004 \pm 186$ (AA81812) AP sin calibrar. Finalmente, una turba pura (PB-9) sella la depositación en el sitio. Recabarren et al. (2011) interpretaron que las turbas fueron depositados en un ambiente pantanoso en un brazo abandonado de un meandro del antiguo río Damas, después de la erosión fluvial de la zona sur de los sedimentos del Pleistoceno, que conforman el área de colinas. La grava fina y media presentes como elementos alóctonos en la turba PB-7 y PB-8 representan la invasión fluvial espóradica de algunas áreas de la ciénaga; PB-9 fue depositado en un pantano sin aporte fluvial.

Entre los años 2007 y 2010 se excavaron un total de $69 \mathrm{~m}^{2}$, recuperándose 912 especímenes óseos de fauna Pleistocena, los que provienen exclusivamente de la capa PB-7. Hasta el momento se han podido identificar representantes de a lo menos ocho familias: Gomphotheriidae, Cervidae, Camelidae, Equidae, Cricetidae, Myocastoridae, Mephitidae y Ursidae, transformándose en uno de los yacimientos finipleistocenos más ricos del centro-sur de Chile (Pino et al., 2013).

\section{Material y métodos}

El material estudiado (NISP: 7, 0,76\% del total) proviene de dos temporadas de excavaciones sistemáticas llevadas a cabo en el yacimiento de Pilauco desde 2007 hasta 2010, a los que se agregan los materiales extraídos de manera asistemática en 1986 cuando el yacimiento fue descubierto. Los restos se encuentran depositados en el Museo Histórico de Osorno, y el Laboratorio de Paleoecología del Instituto de Ciencias Ambientales y 

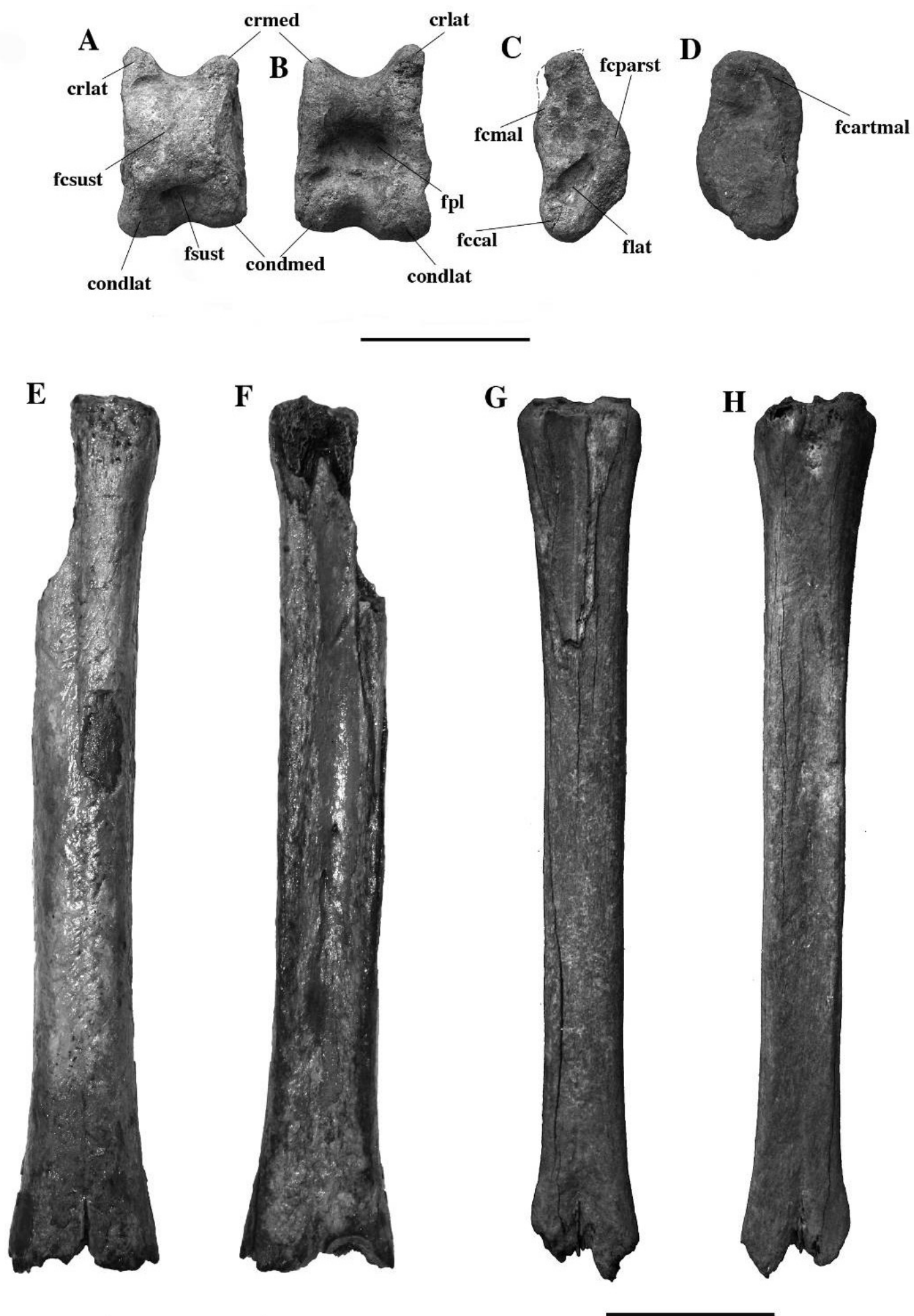

Fig. 3.-cf. Hemiauchenia paradoxa. Astrágalo izquierdo (MHMOP/PI/580), vistas plantar (A), dorsal (B), lateral (C) y medial (D); metacarpo izquierdo (MHMOP/PI/600), vistas dorsal $(\mathrm{E})$ y palmar (F); metatarso derecho (MHMOP/PI/73), vistas dorsal (G) y plantar (H); crmed, cresta medial; crlat, cresta lateral; fcsust, faceta sustentacular; fpl, fosa plantar; condlat, cóndilo lateral; condmed, cóndilo medial; fsust, fosa sutentacular; fcmal, faceta maleolar; fecal, faceta para la porción anterior del calcáneo; flat, fosa lateral; fcparast, faceta parasustentacular; fcartmal, faceta articular para el maléolo medial. La escala mide $5 \mathrm{~cm}$. 
A

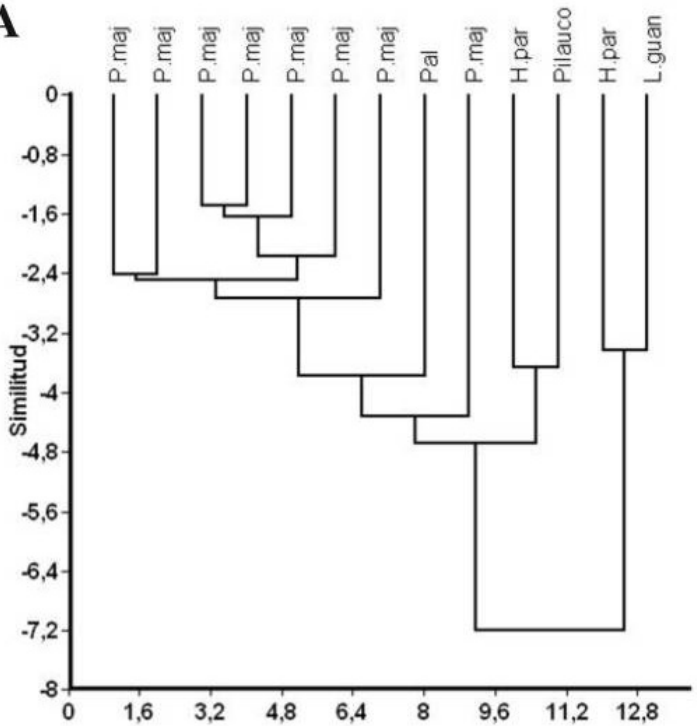

B

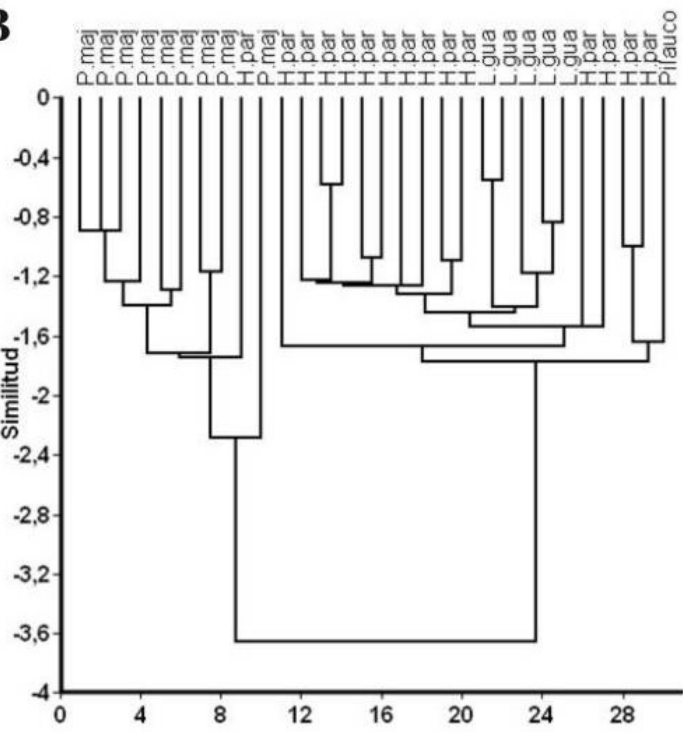

Fig. 4.-Fenogramas (UPGMA) de relaciones de similitud. A Tibia MHMOP/PI/72 y B astrágalo MHMOP/PI/580.

Evolutivas de la Universidad Austral de Chile. Se utilizó material comparativo de camélidos (Lama guanicoe) y cérvidos (Hippocamelus antisensis D'Orbigny, 1834) actuales, depositados en el laboratorio de Zooarqueología del Departamento de Antropología de la Universidad de Chile, colecciones particulares de uno de los autores (Lama guanicoe y Vicugna vicugna) y material fósil del Museo Argentino Bernardino Rivadavia, Buenos Aires y Museo de la Plata, La Plata. La terminología utilizada para la descripción de los materiales se basa en Smust y Bezuidenhout (1987) y Scherer et al. (2007). Los restos óseos fueron medidos siguiendo a Von den Driesch (1976), L'Heureux (2008), Kent (1983), Scherer (2009) y Kaufmann (2009) y los resultados expresados en milímetros. La abreviatura de las medidas utilizadas respeta la nomenclatura original de cada autor. La totalidad de las medidas fueron obtenidas mediante el uso de un calibre digital de $0,01 \mathrm{~mm}$ de precisión y relevadas por un único observador.

Para la asignación específica preliminar de los materiales se llevó a cabo un análisis exploratorio estadístico multivariado. En primer término se realizó un análisis de componentes principales (ACP) a partir de matrices de correlaciones, con el objeto de observar agrupamientos entre los distintos individuos que componen la muestra, utilizando aquellos componentes que explican de mejor manera la varianza del conjunto. De manera complementaria, se empleó el coefíciente de similitud City-Block o Manhattan-Distance, cuyos resultados se volcaron en una matriz de similitud de distancia. A las matrices resultantes se les aplicó un análisis de agrupamiento UPGMA (Unweighted Pair Group Using Arithmetic Averages), resultando un fenograma para cada elemento (Menegaz et al., 1989). Ambas herramientas han sido utilizadas para segregar los distintos representantes de la familia Camelidae (Menegaz et al., 1989; Scherer, 2009; Labarca \& Prieto, 2009). Se utilizaron medidas de referencia de elementos actuales y fósiles a partir de los datos publicados por Scherer (2005) (Tabla 13), Scherer et al. (2007) (Tabla 12), López (2005, Tabla III.7) y de individuos de referencia depositados en el Museo de Historia Natural de San Rafael (Argentina) y en posesión de uno de los autores.

La descripción de las marcas se basa en Behrensmeyer (1978), Lyman (1994), Domínguez-Rodrigo et al. (2009) y Fernández-Jalvo \& Andrews (2003). Las fracturas se describen en función de Johnson (1983) y Ono (2005). Las huellas fueron observadas con lupas de bajo aumento (30x). El estudio tafonómico que se presenta en este trabajo es parte de un análisis mayor el cual se encuentra actualmente en proceso y que se encuentra parcialmente publicado en Pino et al. (2013).

Abreviaturas métricas: Gl: Largo máximo (CT sensu Scherer, 2009), GB: Ancho máximo (Lmax sensu Scherer, 2009), Dl: Profundidad máxima lat- 
eral (Emax sensu Scherer, 2009), CTT: Largo de la tróclea tibial, Bp: Ancho proximal (LTT sensu Scherer, 2009), LC: Ancho lateromedial del cuello del astrágalo, SDl: Profundidad mínima lateral del astrágalo (EC sensu Scherer, 2009), Bd: Ancho distal (LTN para el astrágalo y LD para la tibia sensu Scherer, 2009), ETN: Profundidad de la tróclea navicular del astrágalo, Dd: Profundidad máxima de la epífisis distal (ED sensu Scherer, 2009), GBF: Ancho máximo de la carilla articular distal de la tibia (LSAD sensu Scherer, 2009), SDd: Profundidad mínima de la epífisis distal de la tibia, ESAD: Profundidad de la carilla articular distal de la tibia, Bfal: Ancho de la faceta anterolateral distal de la tibia; Dfal: Profundidad de la faceta anterolateral distal de la tibia, BFpl: Ancho de la faceta posterolateral distal de la tibia, DFpl: Profundidad de la faceta posterolateral distal de la tibia; SLC: largo menor del cuello de la escápula, Bg: Ancho de la cavidad glenoidea; Lg: Largo de la cavidad glenoidea: Glp: largo máximo del proceso articular; SCA169: Ancho cráneo-lateral del proceso de la cavidad glenoidea; SBI: Ancho mínimo del cuello del isquion, La: largo del acetábuo, incluyendo el borde; Lar: largo del acetábulo en el borde; PELV171: Ancho diagonal acetabular, PELV174: Ancho diagonal a la altura del surco ilíaco ventral, PELV175: Ancho mínimo de la escotadura acetabular; DmdC: Diámetro mesiodistal del canino; DblC: Diámetro bucolingual del canino.

Abreviaturas institucionales: MHMOP. Colección de paleontología, Museo Histórico Municipal de Osorno.

\section{Paleontología sistemática}

Orden Cetartiodactyla Montgelard et al. (1997)

Suborden Tylopoda Illiger, 1811

Familia Camelidae Gray, 1821

Subfamilia Camelinae Zittel, 1893

Tribu Lamini Webb, 1974

Hemiauchenia Gervais \& Ameghino, 1880

cf. Hemiauchenia paradoxa Gervais \& Ameghino, 1880

(Figuras 2 y 3 Tablas 1-5)

Material: Incisivo/incisivo caniniforme (MHMOP /PI/540); escápula izquierda (MHMOP/PI/602); metacarpo izquierdo (MHMOP/PI/600); coxal derecho (MHMOP/PI/601); tibia distal derecha (MHMOP/PI/72); astrágalo izquierdo (MHMOP /PI/580); metatarso derecho (MHMOP/PI/73), provenientes de la capa $\mathrm{Pb}-7$ del yacimiento de Pilauco.

Descripción: La pieza MHMOP/PI/540 corresponde a un incisivo caniniforme o bien a un incisivo de pequeñas dimensiones (Figura 2D). Posee una forma semilunar, comprimido labio-lingualmente, se encuentra curvado hacia atrás y presenta una sección ovoidal. La raíz se observa prácticamente completa, a excepción de una pequeña porción del ápice. La corona, por su parte, se encuentra escasamente representada debido al avanzado desgaste por uso, pudiéndose observar la dentina. Si bien la familia Camelidae presenta un casi nulo dimorfismo sexual (Raedeke, 1978), el tamaño de los caninos o incisivos caniniformes ha sido utilizado para separar ambos sexos (Kauffman, 2009).

La escápula MHMOP/PI/602 se encuentra prácticamente completa, a excepción del segmento proximal y la porción proximal lateral de la espina (Figura $2 \mathrm{~A}-\mathrm{C}$, Tabla 2). La hoja posee en términos generales una morfología triangular, corriendo su espina en sentido proximal-distal por la cara lateral, aunque emplazada en el tercio craneal. Lo anterior genera que ambas fosas presenten tamaños muy distintos, ya que la fosa supraespinosa es comparativamente mucho más angosta que la infraespinosa. Esta última es de fondo plano y se encuentra flanqueada en su porción caudal por el borde de la hoja, la que se proyecta en sentido lateral. Este borde es comparativamente más robusto y termina en una cara plana, a diferencia del borde craneal que efectivamente termina en un borde redondeado. La cara lateral de la hoja es levemente cóncava debido a la presencia de los bordes craneales y caudales. El cuello de la escápula corresponde al sector más angosto de la pieza, ya que éste, hacia distal, da paso a la cavidad glenoidea, al tubérculo supraglenoideo y el proceso coracoides. Sobre el cuello se proyecta la parte distal de la espina la que remata en el acromion, el que presenta el ápice fracturado, aunque retiene hasta la altura del inicio del tubérculo supraglenoideo. La cavidad supraglenoidea es cóncava y presenta morfología circular; en su borde craneal presenta una pequeña escotadura semicircular. El tubérculo es robusto, bien marcado, proyectándose hacia medial hasta dar paso al proceso coracoides, el que conforma una pequeña protuberancia comparativamente menos desarrollada.

La pieza MHMOP/PI/600 corresponde a la porción diafisaria de un metacarpo izquierdo (Figura 3D-E). Su cara dorsal presenta sección cóncava pudiéndose apreciar en su sector medial una marca- 
da ranura que recorre longitudinalmente toda la pieza, la que corresponde al punto de unión de los dedos III y IV. Hacia el extremo distal, este accidente da paso a una incisura intertroclear bien marcada y profunda, la que sin embargo se encuentra incompleta debido a una fractura de morfología aserrada. Por lo anterior, los cóndilos articulares se encuentran ausentes, aunque es posible apreciar el comienzo de sus respectivos cuerpos. Por su parte, la cara palmar presenta una sección convexa en su sector proximal y medial, debido a la presencia de un profundo surco longitudinal, el que se encuentra flanqueado por dos marcados rebordes. En sector medial de la cara palmar es posible apreciar dos agujeros nutricios dispuestos casi en forma paralela, separados entre sí por cerca de $12 \mathrm{~mm}$. En el sector proximal de la pieza sólo es posible advertir el dígito III, el que aún retiene parte de la carilla articular para los carpos segundos y terceros.

El coxal MHMOP/PI/601 retiene el cuello del ilion, acetábulo completo, el segmento craneal del pubis y el cuerpo del isquion hasta la incisura isquiática menor (Figura 2E-F, Tabla 3). El cuello del ilion se encuentra comprimido lateromedialmente, generando un marcado borde por su cara ventral. Por su cara lateral, muy próximo al acetábulo se ubica una depresión ovoidal que corresponde a la inserción del músculo recti femoris. El acetábulo presenta una carilla lunar continua y semicircular, terminando en una incisura acetabular ancha y bien marcada. La fosa acetabular, por su parte, presenta una morfología rectangular, con dirección lateromedial, y una sección levemente cóncava, encontrándose mucho más proyectada hacia lateral. El acetábulo presenta un pequeño reborde que se encuentra mejor representado en los extremos de la carilla lunar. El isquion presenta sección rectangular, aplanada dorsolateral-ventromedialmente; hacia dorsal se observa la espina isquiática, la que hacia caudal continúa en la incisura isquiática menor, la que se conforma a partir de una pequeña escotadura, la que se encuentra fracturada. El pubis se encuentra fracturado en el cuello, observándose una sección subrectangular comprimida dorsoventralmente.

El espécimen MHMOP/PI/72 corresponde a la porción distal de una tibia derecha completa, la que mantiene la cóclea tibial y parte de la diáfisis distal (Figura G-2, Tabla 4). La parte craneal de ésta es levemente cóncava, destacando rugosidades y una hendidura para la inserción muscular en su costado lateral. En éste es posible apreciar el inicio del surco maleolar, el que se manifiesta inicialmente como una leve acanaladura de sección subcuadrangular, hasta terminar en una escotadura de sección subcircular. La superficie articular distal destaca por tres rasgos anatómicos: la cóclea tibial, el maléolo medial y la faceta articular para el maléolo lateral. La cóclea tibial presenta dos grandes escotaduras dispuestas de forma casi paralela entre sí en sentido antero-posterior, las que articulan con la tróclea proximal del astrágalo. Estas facetas, en Equidae se encuentran dispuestas en forma oblicua. Ambas carillas articulares presentan una morfología relativamente ovalada, aunque la faceta medial presenta su pared medial comparativamente más recta debido a la presencia del maléolo medial. Por su parte, la faceta lateral posee una morfología levemente más redondeada, lo que se traduce en un ancho mayor en sentido latero-medial. Ambas se encuentran separadas por una pequeña cresta sagital de superficie redondeada, la que aparece delimitada en sentido antero-posterior por dos procesos (anterior y posterior). El proceso anterior es más grande en sentido próximo-distal y posee una forma similar a un triángulo rectángulo. Las carillas articulares para el maléolo lateral son redondeadas, siendo la carilla anterior más pequeña que la posterior. Éstas se encuentran separadas por un profundo surco para el proceso proximal del maléolo lateral. Por último, el maléolo medial, ubicado en el borde medio-lateral de la cóclea, corresponde a un proceso robusto, proyectado distalmente (aunque menos que el proceso anterior) y orientado hacia anterior. En Cervidae este proceso es comparativamente más angosto y puntiagudo y corresponde al punto más distal de la tibia. En vista anterior, el surco que se genera entre el maléolo y el proceso anterior es poco profundo; en Cervidae en cambio, este accidente posee una marcada profundidad, alcanzando, en sentido distal-proximal, hasta el inicio de la faceta lateral de la cóclea.

$\mathrm{El}$ astrágalo $\mathrm{MHMOP} / \mathrm{PI} / 580$ presenta un aspecto rectangular (Figura 3A-C, Tabla 5). La tróclea proximal para la articulación con la cóclea tibial presenta dos crestas principales (medial y lateral), las que se disponen paralelas entre sí, longitudinales en relación al eje del hueso y separadas por una escotadura de morfología semicircular. La cresta medial es más pequeña que la lateral (menos desarrollada en sentido proximal). Esta tróclea se encuentra separada de la faceta sustentacular por un pequeño surco que se dispone de manera transversal al eje del hueso, mientras que por su cara dorsal alcanzaría 
A

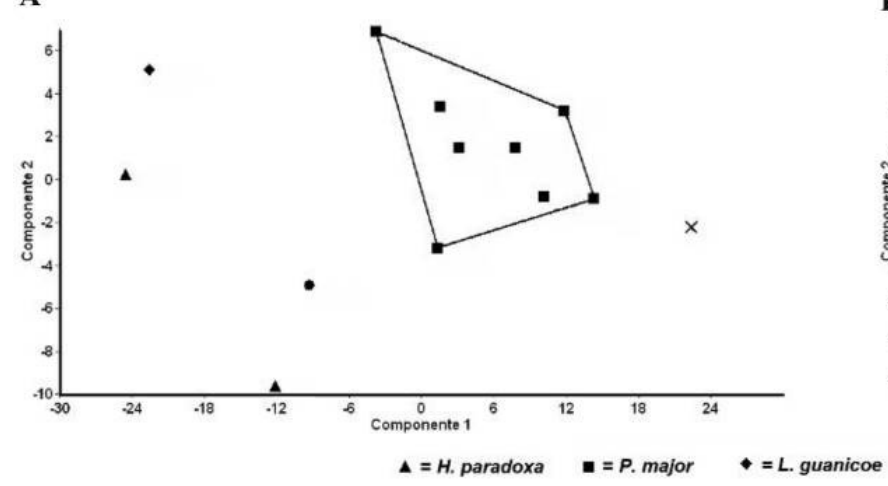

B

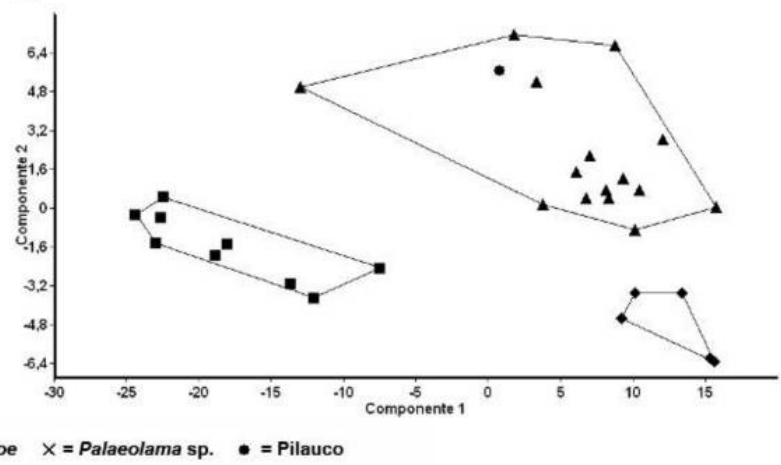

Fig. 5.-Gráficos de componentes principales. A Tibia MHMOP/PI/72 y B astrágalo MHMOP/PI/580.

hasta la fosa dorsal, cuestión que no puede ser advertida con claridad debido a la erosión de la pieza. En Cervidae, la cresta lateral presenta una pequeña curvatura hacia lateral, cuestión que no ha sido advertida en este espécimen. La tróclea distal, presenta a su vez dos facetas articulares o cóndilos de morfología semicircular, separados por una pequeña cresta sagital. El cóndilo medial, que articula con el tarso central, es comparativamente más ancho latero-medialmente que el cóndilo medial, presentando además, una pequeña depresión. Por su cara plantar destaca la faceta sustentacular, la que presenta una morfología rectangular y sección relativamente convexa próximo-distalmente. En este caso, la porción lateral presenta un mayor desarrollo en sentido dorso-plantar que aquella medial. Bajo ésta se ubica la fosa subsustentacular, de sección oval, la que a su vez se conecta con la tróclea distal. Por su parte dorsal, en el sector central se ubica la fosa dorsal, de morfología oval. La cara medial se encuentra erosionada, pudiéndose apreciar, en su sector proximal, parte de la faceta articular para el maléolo medial de la tibia. En Cervidae esta carilla se extiende hasta alcanzar la cresta lateral por su cara plantar, cuestión que no se observa en el espécimen en estudio. Una situación similar ocurre con la cara lateral, la que presenta la faceta parasustentacular erosionada y la faceta maleolar parcialmente fracturada. Aquí sólo es posible observar, en su parte distal, la fosa lateral, de morfología ovoidal.

El metatarso MHMOP/PI/73 exhibe fundamentalmente su porción diafisaria, en el que es posible observar la fusión de los metápodos III y IV en casi toda su longitud (Figura 3F-G). La parte proximal aún mantiene porciones de las facetas articulares para el tercer y cuarto tarsiano, aunque estas se encuentran muy incompletas. Sólo es posible mencionar que entre ambas se observa una pequeña fosa subcircular, la que se proyecta levemente hacia plantar. La cara dorsal de la diáfisis presenta sección cóncava, mientras que su cara plantar se observa comparativamente convexa debido a la presencia de un amplio surco que se dispone en su parte central y que recorre prácticamente toda su extensión longitudinalmente hasta poco antes de la separación de las carillas articulares distales. En el sector medio de la cara plantar, exhibe dos agujeros nutricios separados entre sí por cerca de $20 \mathrm{~mm}$ los que ubican de forma oblicua uno del otro. Hacia la parte distal de la pieza es posible observar el inicio de la incisura intertroclear, la que permite la separación de los respectivos cóndilos, los que en este caso se encuentran ausentes.

\section{Tafonomía}

Las piezas descritas presentan diversas marcas en su superficie, las que permiten reconstruir la historia tafonómica de los restos de Lamini en el yacimiento. En términos generales, el conjunto presenta un buen estado de preservación, sin signos de meteorización, aun cuando cuatro especímenes presentan abrasión sedimentaria, en grados medio y severo (sensu Gutiérrez \& Kaufmann 2007) lo que afectó las capas superficiales del hueso, exponiendo el tejido esponjoso en algunos casos y eliminando y/o reduciendo accidentes anatómicos. Dentro de este grupo se ubica el metacarpo MHMOP/PI/601 y metatarso 
MHMOP/PI/73. Particularmente este último en su porción próximo-lateral y plantar, exhibe parte del tejido esponjoso en algunos sectores acotados, en donde se han depositado sedimentos arenosos incluidos en la matriz orgánica. Una situación similar ocurre con el astrágalo MHMOP/PI/580, el que presenta una clara erosión diferencial, la que se manifiesta fundamentalmente en crestas y promontorios, y en menor medida en surcos y fosas (Figura $3 \mathrm{~A}-\mathrm{C})$. $\mathrm{Al}$ igual que en la pieza $\mathrm{MHMOP} / \mathrm{PI} / 73$, en algunos sectores se aprecia tejido esponjoso en donde se observan restos de sedimentos arenosos.

Un segundo tipo de marcas registradas en el conjunto óseo corresponden a negativos de raíces (Lyman, 1994). Éstas se generan debido a la secreción de ácido húmico, el que disuelve la porción del hueso que se encuentra en contacto con estas estructuras (Lyman, 1994). Regularmente generan marcas poco profundas y con un patrón dendrítico. Este tipo de modificaciones afecta al metatarso MHMOP/PI/73, metacarpo MHMOP/PI/61 y tibia MHMOP/PI/72 (Figura 6C).

Por último, tres piezas (escápula MHMOP/ PI/602, tibia MHMOP/PI/72 y metatarso MHMOP/PI/73) exhiben marcas de pisoteo (Figura $6 \mathrm{~A}$ y B). Éstas se ubican fundamentalmente en las prominencias, se caracterizan por ser muy superficiales, de lados irregulares, levemente sinuosas y con orientaciones azarosas; algunas de éstas confluyen incluso formando ángulos de $90^{\circ}$. La gran mayoría de las marcas no presentan estriaciones internas, y las que sí las exhiben, se caracterizan por ser irregulares y discontinuas (DomínguezRodrigo et al., 2009; Olsen \& Shipman, 1989), descartando así eventuales huellas de corte por acción de instrumentos humanos.

Dentro del conjunto se registran fracturas con diversas morfologías y ángulos, las que son indicativas del estado del hueso al momento de su rompimiento (Johnson, 1983; Ono, 2005). De esta manera, la porción distal de tibia, exhibe una fractura fresca, debido a la presencia de bordes de fractura suave y a la línea de fractura sinuosa (Johnson, 1983; Outram, 2003). No es posible apreciar negativos de impacto, lascados u otro rasgo que permita sugerir la presencia de una carga dinámica (percusión) en la diáfisis (Johnson, 1983). El resto de los huesos largos (metatarso y metacarpo) presentan una combinación de fracturas incluyendo borde aserrado o de líneas rectas (columnar) (Lyman, 1994). Tanto el metatarso como el coxal presentan agrietamientos y líneas de fisura.

\section{Discusión}

\section{Taxonomía}

El estudio monográfico de Scherer (2009) sobre la Tribu permitió definir de mejor manera los caracteres diagnósticos de cada una de las formas reconocidas para el clado. Gran parte de los rasgos diagnósticos se ubican en el cráneo, particularmente en los molares, o se desprenden de análisis osteométricos a partir de unidades completas, fundamentalmente huesos largos. Si bien dentro de los fósiles del yacimiento de Pilauco no se registran los restos señalados anteriormente, desarrollamos un análisis osteométrico multivariado exploratorio con dos elementos postcraneales (tibia distal y calcáneo). Esto, con el objeto de discutir la posibilidad de establecer diferencias métricas taxonómicamente válidas con porciones de elementos o con huesos que tienen más posibilidades de recuperarse completos en yacimientos secundarios, como el analizado aquí. El análisis de coeficientes de similitud efectuado con cuatro medidas de la tibia distal (Figura 4A) permite segregar dos grupos. El primero, de mayor tamaño, incluye a todos los elementos de Palaeolama major además de Palaeolama sp. de Quereo, un elemento de Hemiauchenia paradoxa y el especímen de Pilauco. El grupo pequeño asocia a Lama guanicoe y un individuo de Hemiauchenia paradoxa. Dentro del primer conjunto, la tibia MHMOP/PI/72 se agrupa con H. paradoxa. El ACP, por su parte, entrega resultados similares (Figura $5 \mathrm{~A}$ ), ya que el primer componente (que explica $87,962 \%$ de la varianza), separa cuatro grupos, asociándose el espécimen de Pilauco con H. paradoxa grande. Estos resultados permiten confirmar que los representantes del género Palaoelama corresponden a los de mayor porte dentro de los Lamini.

En lo que respecta a los astrágalos, se consideraron ocho medidas. Esta vez, el dendrograma obtenido separa con claridad a Palaolama major, de mayores dimensiones, de los restantes Lamini incluidos en el análisis. Dentro del grupo "pequeño", compuesto por H.paradoxa + L.guanicoe + Pilauco, los guanacos actuales forman un subconjunto bien definido, mientras que la pieza MHMOP/PI/580 se agrupa con los representantes de $H$. paradoxa. El ACP, en este caso, permite segregar de mejor manera la muestra estudiada, pudiéndose apreciar tres claros conjuntos de medidas, los que coinciden con las formas de referencia (P. major, H. paradoxa y L. guanicoe). El espécimen 


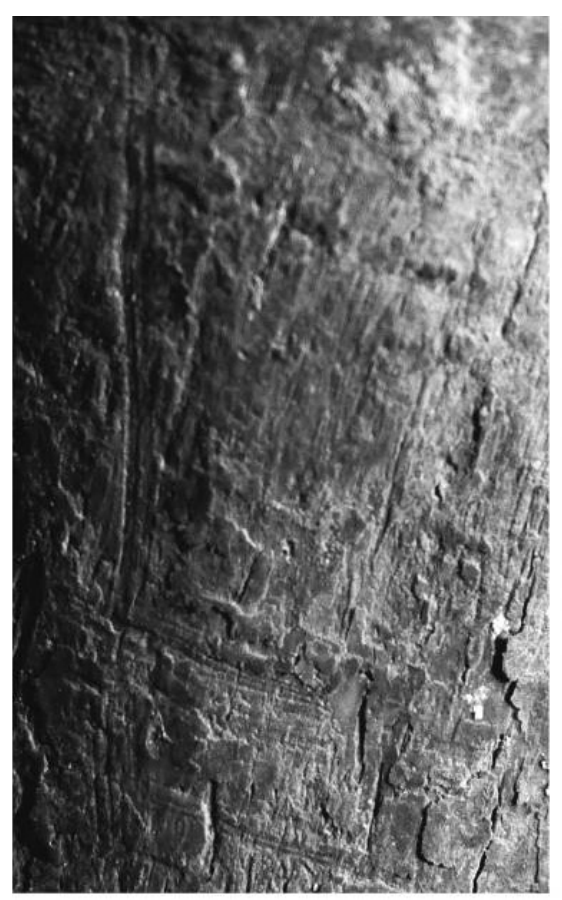

A

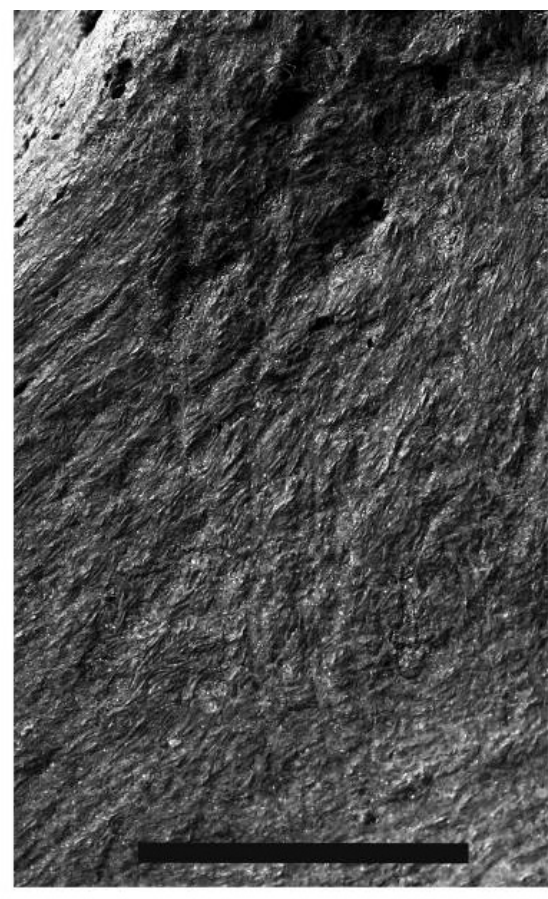

B

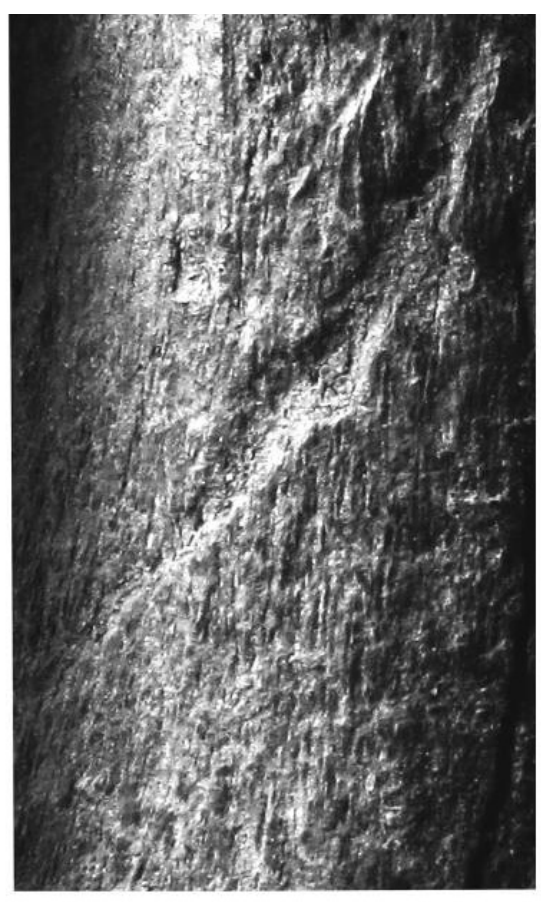

C

Fig. 6.-Marcas en los huesos. A marcas de pisoteo (MHMOP/PI/602), B (MHMOP/PI/602) y C (MHMOP/PI/72) marcas de raíces. La escala mide $0,5 \mathrm{~mm}$.

de Pilauco se ubica dentro de la variabilidad métrica de $H$. paradoxa. Por otro lado, es posible apreciar una gradiente de tamaño, en donde $H$. paradoxa se ubica en una posición intermedia entre $P$. major y $L$. guanicoe.

Debido a la ausencia de elementos morfológicamente diagnósticos y considerando sólo los resultados osteométricos, los materiales del yacimiento de Pilauco son asignados a cf. Hemiauchenia paradoxa, a la espera de nuevos materiales fósiles que permitan contrastar esta asignación preliminar.

\section{Tafonomía}

Producto de la ausencia de oxigeno en la turba PB-7 no es esperable reconocer evidencias de meteorización. A nuestro juicio, las marcas presentes en la muestra analizada son coherentes con la composición de la capa PB-7 y con el ambiente de inundación fluvial esporádico inferido para ésta, dado que no se reconocen estratos o láminas que indiquen depositación exclusivamente fluvial. De esta manera, las marcas de pisoteo se habrían origi- nado por el contacto del hueso con los componentes arenosos y rudíticos mezclados con la turba de la capa, ya sea a través de la acción in situ sobre especímenes previamente depositados o por la presión ejercida contra éstos por parte de animales. El hecho de que se registren huellas de pisoteo en huesos frágiles como la hoja de la escápula confirma la presencia de sedimentos blandos y plásticos, los que habrían absorbido a los materiales fósiles, evitando así su fractura y favoreciendo su sepultación. Este proceso explicaría, además, la ausencia de elementos meteorizados.

Por su parte, las marcas de abrasión habrían sido producidas tanto por el pisoteo, como por el contacto sistemático del hueso con los sedimentos arenosos desplazados por la acción hídrica (Olsen \& Shipman 1989; Fernández-Jalvo \& Andrews, 2003), los que habrían operado in situ sobre las unidades anatómicas ya depositados en la matriz orgánica del pantano (Gutiérrez \& Kaufmann, 2007) o bien, durante el traslado de las piezas por la acción hídrica, especialmente aquellas de morfología redondeada como la pieza MHMOP/PI/580. Estudios experimentales llevados a cabo con restos 
óseos de diversos mamíferos medianos y grandes (Ovis aries Linnaeus, 1775, Canis latrans Say, 1823 y Lama guanicoe) apuntan a que los huesos con formas redondeadas son comparativamente más susceptibles de ser transportados por rodadura en un ambiente fluvial que aquellos planos (Voorhies, 1969; Gutiérrez \& Kaufmann, 2007; Lyman, 1994; Frostick \& Reid, 1983).

La presencia de modificaciones llevadas a cabo por raíces permite postular un ambiente sedimentario capaz de soportar vegetación (Lyman, 1994), al tiempo que es un indicador de estabilidad relativa de los huesos al interior de la matriz sedimentaria, tal como en el caso de un pantano. En el sitio han sido descritas la presencia de esporas de plantas acuáticas y helechos (Abarzúa \& Gajardo-Pinchicura, 2008). En dos casos (metatarso y metacarpo), no obstante, se aprecian marcas de abrasión y raíces, lo que permitiría postular dos escenarios depositacionales sucesivos operando sobre los huesos.

Dado que no es posible apreciar rasgos que permitan sugerir la presencia de una carga dinámica (percusión) en las diáfisis (Johnson, 1983), la fractura fresca de borde sinuoso del espécimen MHMOP/PI/72 probablemente se habrían generado a través del pisoteo de los especímenes, cuestión apoyada por la presencia constante de marcas atribuidas a este proceso tafonómico. Por su parte, las fracturas de líneas rectas o columnares fueron producidas en estado no fresco (Villa \& Mahieiu, 1991; Lyman, 1994; Outram, 2003). Los agrietamientos y líneas de fisura del metatarso como el coxal podrían interpretarse como producto de la rápida desecación de las piezas una vez extraídas de la matriz.

En suma, es posible proponer a partir exclusivamente de las marcas observadas en los huesos de Lamini, la presencia de tres eventos dentro de la historia tafonómica de los fósiles; abrasión por partículas sedimentarias (in situ o a través de transporte fluvial), pisoteo y acción de raíces. No se trata de procesos diacrónicos, ya que por ejemplo, la abrasión y el pisoteo podrían haber afectado simultáneamente a los restos óseos. Todas las marcas son coherentes con la matriz sedimentaria del depósito y con el contexto depositacional inferido para los restos óseos, donde el desborde del cauce principal del río habría tenido una competencia de energía moderada a leve. La presencia diferencial de las modificaciones estudiadas en la colección no permite asegurar que se trate de un solo individuo, pudiendo corresponder a distintos eventos depositacionales, no descartándose eventuales movimientos por la acción del agua, particularmente en las piezas más pequeñas. Si bien la muestra analizada es escasa como para ser representativa de todos los eventos postdepositacionales ocurridos en el yacimiento, las modificaciones descritas en el presente trabajo son coherentes con aquellas observadas en otros materiales fósiles de Pilauco (Pino et al., 2013).

\section{Consideraciones biogeográficas y paleoecológicas}

Webb \& Stehli (1995) sugieren que una forma ancestral de Hemiauchenia habría emigrado hacia Sudamérica en el Plioceno final, dando origen posteriormente a la única forma reconocida para Sudamérica, H. paradoxa (Webb \& Stehli, 1995; Scherer, 2009). Los primeros registros de este taxón provienen del Marplatense (Plioceno final Pleistoceno temprano) de la región pampeana, aumentando considerablemente su distribución hacia el Pleistoceno final, extendiéndose hasta el sur de Bolivia por norte (circa $21^{\circ} \mathrm{S}$ ) y la región patagónica central por el sur $\left(48^{\circ} \mathrm{S}\right)$ (Scherer, 2009; Paunero et al., 2007; Marshal et al., 1984). Su presencia en el yacimiento de Pilauco, por tanto, es coherente con la distribución latitudinal del género. Se trataría, no obstante, del primer registro al Oeste de la cordillera de los Andes, lo que obliga a discutir eventuales rutas de dispersión. Al respecto, Casamiquela (1968), a partir de la presencia del género Glossotherium Owen, 1839 en la región de Lonquimay (circa $38^{\circ} \mathrm{S}$ ), sugiere rutas de dispersión de faunas orientales a través de corredores transandinos de baja altura, durante momentos interestadiales. Esta hipótesis es apoyada por Moreno et al. (1994) a partir de la presencia de Stegomastodon Pholig, 1912 en Chile central. Atendiendo a la ubicación del sitio de Pilauco, es posible sugerir una vía de acceso similar, cuestión que deberá ser corroborada con estudios sistemáticos en sectores periandinos.

Hemiauchenia ha sido tradicionalmente asociada a paisajes abiertos con pastizales (Webb \& Stehli 1995), infiriéndose hábitos cursoriales, debido a la presencia de metapodios largos y gráciles, y epipodios más cortos. Esta interpretación ha sido cuestionada por Scherer (2009) debido a que en yacimientos del sur de Bolivia han sido registrados en simpatría $H$. paradoxa y Palaeolama weddelli, este último con metapodios cortos y robustos y de epi- 
podios largos, supuestamente adaptados a ambientes irregulares o rocosos (Scherer, 2009). Estudios de isótopos estables en restos de Hemiauchenia de Florida (EEUU), indican una dieta mixta (pastadora-ramoneadora) con una preferencia por el consumo de árboles y arbustos (Feranec, 2003). Los estudios palinológicos efectuados en el sitio Pilauco sugieren un ambiente abierto con presencia de bosque en las proximidades, debido a la predominancia de Poaceae y Asteraceae en el registro, y en menor medida, de especies propias del bosque Norpatagónico (e.g. Nothofagus dombeyi, Podocarpus nubigenus, Fitzroya sp.) (Abarzúa \& Gajardo-Pilchicura, 2008). Estos resultados serían coherentes con la presencia de $H$. paradoxa en el registro del yacimiento, así como de otros taxones adaptados a ambientes abiertos (e.g. Stegomastodon, Equus (A.) sp.).

\section{Conclusiones}

Se constata la presencia de la tribu Lamini en el yacimiento paleontológico de Pilauco, siendo asignados los restos provisoriamente a cf. Hemiauchenia paradoxa, sobre la base de análisis osteométricos multivariantes debido a la ausencia de elementos morfológicamente diagnósticos, los que se ubican fundamentalmente en la región craneal (Scherer et al., 2007; Scherer, 2009). Las excavaciones sistemáticas en el yacimiento se encuentran aún en curso, por lo que es posible esperar nuevos materiales de Camelidae, que permitan confirmar o descartar la asignación taxonómica presentada.

El estudio tafonómico efectuado permite inferir una variada historia depositacional para el yacimiento, en donde los restos fósiles se habrían visto expuestos a distintos agentes naturales. Se identificaron marcas de raíces, abrasión por acción hídrica y marcas de pisoteo, las que son coherentes con la composición de la capa portadora de los fósiles, así como de las condiciones geomorfológicas definidas para ésta. No es posible descartar el traslado y/o reacomodo de algunas piezas por los agentes fluviales, particularmente aquellas de menor tamaño y morfología redondeada. No se aprecian marcas de origen cultural en los restos analizados.

Hipotéticamente se postula el ingreso de cf. $H$. paradoxa desde el área de Pampa/Patagonia Argentina, a través de corredores transandinos de baja altura, como ha sido sugerido para otros taxones presentes en el Pleistoceno de Chile. El paisaje abierto inferido para el yacimiento de Pilauco hacia el Pleistoceno tardío, sería coherente con los requerimientos ambientales postulados para este taxón.

\section{AGRADECIMIENTOS}

Agradecemos a Isabel Cartajena (FACSO- Universidad de Chile) por la posibilidad de utilizar los materiales comparativos de cérvidos y camélidos. A Lucas Pomi y Marcelo Reguero (MLP) y Alejandro Kramarz (MACN) por la posibilidad de revisar las colecciones fósiles depositadas en el Museo de la Plata, La Plata y en el Museo Argentino Bernardino Rivadavia, Buenos Aires, respectivamente. A Clara Otaola (CONCIET-Museo de Historia Natural de San Rafael) por las medidas de guanacos proporcionadas. Esta contribución fue financiada parcialmente por el proyecto FONDECYT 1100555. Un revisor anónimo y María Teresa Alberdi (Departamento de Paleobiología, Museo Nacional de Ciencias Naturales, CSIC) colaboraron a mejorar sustancialmente una primera versión de este trabajo.

\section{Referencias}

Abarzúa, A. \& Gajardo-Pinchicura, A. (2008) ¿Y qué nos cuentan los polen?: La historia climática y vegetacional del sitio Pilauco. In: Pilauco: Un sitio complejo del Pleistoceno tardio. Osorno, Norpatagonia chilena (Pino, M., ed.). Imprenta América, Valdivia, Chile, 4954, $105 \mathrm{pp}$.

Behrensmeyer, A. K. (1978). Taphonomic and ecologic information from bone weathering. Paleobiology 4 , 150-62.

Borrero, L.A.; Martin, F.M. \& Prieto, A. (1997). La Cueva Lago Sofía 4, Úlima Esperanza: una madriguera de felino del Pleistoceno tardío. Anales del Instituto de la Patagonia, Serie Ciencias Humanas, 25: 103-122.

Cartajena, M.I.; López, P. \& Martínez, I. (2010). New camelid (Artiodactyla: Camelidae) record of the late Pleistocene of Calama (Second Region, Chile): a morphological and morphometric discussion. Revista Mexicana de Ciencias Geológicas, 27: 197-212.

Casamiquela, R. (1968). Noticia sobre la presencia de Glossotherium (Xenartra, Mylodontidae) en Chile central. Anales del Museo de Historia Natural de Valparaiso, 1: 143-172.

Casamiquela, R. (1999). The Pleistocene vertebrate record of Chile. Quaternary of South America and Antarctic Peninsula, 7: 91-107.

Cione, A.L. \& Tonni, E. P. (1995). Chronoestratigraphy and 'Land Mammals-Ages': The Uquían Probleam. Journal of Paleontology, 69: 135-159.

Domínguez-Rodrigo, M.; De Juan, S. \& Galán, A.B. (2009). A new protocol to differentiate trampling marks from butchery cut marks. Journal of Archaeological Science, 36: 2643-2654. doi:10.1016/ j.jas.2009.07.017 
Feranec, R.S. (2003). Stable isotopes, hypsodonty, and the paleodiet of Hemiauchenia (Mammalia: Camelidae): A morphological specialization creating ecological generalization. Paleobiology, 29: 230242. doi: $10.1666 / 0094-8373(2003) 029<0230$ : SIHATP $>2.0 . \mathrm{CO} ; 2$

Fernández-Jalvo, Y. \& Andrews, P. (2003). Experimental effects of water abrasion on bone fragments. Journal of Taphonomy, 1: 147-163.

Frostick, L. \& Reid, I. (1983). Taphonomic significance of subaerial transport of vertebrate fossils on steep semi-arid slopes. Lethaia, 16: 157-164. doi:10.1111/j.1502-3931.1983.tb01711.x

González, B.A.; Palma, R.E.; Zapata, B. \& Marín, J.C. (2006). Taxonomic and biogeographic status of guanaco Lama guanicoe (Artiodactyla, Camelidae). Mammal Review, 36: 157-178. doi:10.1111/j.1365-2907.2006. 00084.x

Gutiérrez, M. \& Kaufmann, C. (2007). Criteria for the identification of formation process in guanaco (Lama guanicoe) bone assemblages in fluvial-lacustrine environments. Journal of Taphonomy, 5: 151-176.

Johnson, E. (1983). A framework for Interpretation in Bone Technology. In: Carnivores, Human Scavengers and Predators: A Question of bone technology (LeMoine, G. M. \& MacEarchen, A. S., eds.). University of Calgary Archaeological Association, 55-93.

Kaufmann, C.A. (2009). Estructura de edad y sexo en guanaco: estudios actualísticos y arqueológicos en Pampa y Patagonia. Sociedad Argentina de Antropología, Buenos Aires, 315 pp.

Kent, J.D. (1982). The Domestication and exploitation of the South American camelids: methods of analysis and their application to circumlacustrine archaeological sites in Bolivia and Peru. Ph. D. dissertation. Departament of Anthropology, Washington University. St. Louis, Missouri, USA. University Microfilms International, Ann Harbor, Michigan.

Labarca, R. \& López, P. (2006). Los mamíferos finipleistocénicos de la Formación Quebrada Quereo: (IV Región-Chile): biogeografía, bioestratigrafía e inferencias paleoambientales. Mastozoología Neotropical, 13: 89-101.

Labarca, R. \& Prieto, A. (2009). Osteometría de vicugna vicugna Molina, 1782 en el Pleistoceno final de Patagonia meridional chilena: Implicancias paleoecológicas y biogeográficas. Revista del Museo de Antropología, 2: 127-140.

Latorre, C. (1998). Paleontología de mamíferos del Alero Tres Arroyos 1, Tierra del Fuego, XII Región, Chile. Anales del Instituto de la Patagonia, Serie Ciencias Naturales, 26: 77-90

L'Heureux, G.L. (2008). El estudio arqueológico del proceso coevolutivo entre las poblaciones humanas y las poblaciones de guanaco en Patagonia meridional y norte de Tierra del Fuego. BAR International Series 1751, Oxford., 275 pp.

López, P. (2005). Tafonomía en la costa meridional del norte semiárido de Chile (IV región). Alcances cultu- rales y paleoecológicos hacia el Pleistoceno final en la Comuna de Los Vilos $\left(31^{\circ}\right.$ latitud S). Tesis de pregrado. Departamento de Ciencias Sociales de la Universidad de Chile, Santiago, Chile, 215 pp.

López, P. (2007). Tafonomía de los mamíferos extintos del Pleistoceno tardío de la costa meridional del semiárido de Chile (IV Región-32 ${ }^{\circ}$ latitud S). Alcances culturales y paleoecológicos. Chungara 39: 69-86.

López, P.; Jackson, D. \& Jackson, D. (2005). Presencia del género Palaeolama Gervais (Artiodactyla, Camelidae) en el extremo meridional del semiárido de Chile. Boletín del Museo de Historia Natural, Chile, 54: 129-140.

Lyman, R.L. (1994). Vertebrate Taphonomy. Cambridge: Cambridge University Press. 576 pp.

Marshall, L.G. \& Sempere, T. (1991). The Eocene to Pleistocene vertebrates of Bolivia and their stratigraphic context: a review. Revista Técnica de Yacimientos Petroliferos Fiscales Bolivianos, 12: 631-652.

Marshall, L.G.; Berta, A.; Hoffstetter, R.; Pascual, R.; Bombin, M. \& Mones, A. (1984). Mammals and stratigraphy: geochronology of the continental mammalbearing Quaternary of South America. Paleovertebrata, Mémoire Extraordinaire, 1-76.

MacFadden, B. \& Shockey, B. (1997). Ancient feeding ecology and niche differentiation of Pleistocene mammalian herbivores from Tarija, Bolivia: morphological and isotopic evidence. Paleontology, 23: 77-100.

McKenna, M.C. \& Bell, S.K. (1997). Classification of Mammals Above the Species Level. Columbia University Press, New York, 631 pp.

Menegaz, A. (2000). Los camélidos y cérvidos del cuaternario del sector bonaerense de la Región Pampeana. Tesis de doctorado, La Plata, Argentina, Facultad de Ciencias Naturales y Museo de La Plata, 211 pp.

Menegaz, A. \& Ortiz-Jaureguizar, E. (1995). Los Artiodáctilos. In: Evolución biológica y climática de la Región Pampeana durante los últimos 5 millones de años (Alberdi, M. T.; Leone, G. \& Tonni, E. P. Eds). Un ensayo de correlación con el Mediterráneo occidental, Monografías, Museo Nacional de Ciencias Naturales, CSIC, Madrid, 311-337.

Menegaz, A.; Goin, F. \& Ortíz-Jaureguízar, E. (1989). Análisis morfológico y morfométrico multivariado de los representantes fósiles y vivientes del género Lama (Artiodactyla, Camelidae). Sus implicancias sistemáticas, biogeográficas, ecológicas y biocronológicas. Ameghiniana, 26: 153-172.

Mengoni-Goñalons, G.L. (1987). Modificaciones culturales y animales en los huesos de los niveles inferiores del sitio Tres Arroyos 1 (Tierra del Fuego, Chile). Anales del Instituto de la Patagonia, Serie Ciencias Sociales, 17: 61-66.

Mercer, J.H. (1976). Glacial history of southernmost South America. Quaternary Research, 6: 125-166. doi:10.1016/0033-5894(76)90047-8

Moreno, P.; Villagrán, C.; Marquet, P.A. \& Marshall, L.G. (1994). Quaternary paleobiogeography of northern and central Chile. Revista Chilena de Historia Natural, 67: 487-502. 
Olsen, S.L. \& Shipman, P. (1989). Surface modification on bone: Trampling vs. butchery. Journal of Archaeological Science, 15: 535-553. doi:10.1016/0305-4403 (88)90081-7

Ono, A. (2005). Fracture Patterns of Bone in Archaeological Contexts: Significance of the Casper Site Materials. The Wyoming Archaeologist, 42: 15-48.

Pascual, R. \& Ortíz-Jaureguízar, E. (2007). The Gondwanan and South American episodes: Two major and unrelated moments in the history of the South American mammals. Journal of Mammal Evolution, 14: 75137. doi:10.1007/s10914-007-9039-5

Paunero, R.S.; Albertengo, P.; Cueto, M.; Dávila, A.; Frank, A. \& Olivera, A. (2007). Sitio Casa del Minero 1, localidad arqueológica La María: nuevas evidencias sobre ocupación humana pleistocénica en Santa Cruz. In: Arqueología Argentina en los Inicios de un Nuevo Siglo (Fernando Oiva, Nélida de Grandis, Jorge Rodriguez, eds.). Tomo 1, 337-344.

Pérez, Y.; Milovic, J.; Troncoso, R.; Hanisch, J.; Helms, F. \& Toloczyki, M. (2003). Geología para el ordenamiento territorial: área de Osorno, Región de Los Lagos. Servicio Nacional de Geología y Minería, Carta Geológica de Chile, Serie Geología Ambiental, No. 6, $54 \mathrm{pp}$.

Pino, M.; Chávez.Hoffmeister, M.; Navarro-Harris, X.; Labarca, R. (2013). The late Pleistocene Pilauco site, Osorno, South-central Chile. Quaternary International, doi:10.1016/j.quaint.2012.05.001

Raedeke, K. (1978). El guanaco de Magallanes, Chile. Su distribución y biología. Ministerio de Agricultura de Chile, Corporación Nacional Forestal (CONAF), Chile, Publicación Técnica, 4: 1-182.

Recabarren, O.P.; Pino, M. \& Cid, I. (2011). A new record of Equus (Mammalia: Equidae) from the Late Pleistocene of central-south Chile. Revista Chilena de Historia Natural. 84: 535-542. doi:10.4067/S0716078X2011000400006

Scherer, C.S. (2005). Estudo dos camelidae (Mammalia, Artiodactyla) do Quaternário do Estado do Rio Grande do Sul. Dissertação de Mestrado, Universidade Federal do Rio Grande do Sul. 176.

Scherer, C.S. (2009). Os Camelidae Lamini (Mamma-lia, Artiodactyla) do Pleistoceno da América do Sul: Aspectos taxonômicos e filogenéticos. Tesis Doctoral. Porto Alegre, Brasil, UFRGS - Universidade Federal do Rio Grande do Sul, 460 pp.

Scherer, C.S.; Ferigolo, J.; Ribero, A.M. \& Cartelle, C. (2007). Contribution to the knowledge of Hemiauche- nia paradoxa (Artiodactyla, Camelidae) from the Pleistocene of southern Brazil. Revista brasileira de paleontología, 10: 35-52.

Smuts, M.M.S. \& Bezuidenhout, A.J. (1987). Anatomy of the Dromedary. Clarendon Press, Oxford, 230 pp.

Tauber, A. (1999). Hallazgo de una vicuña en el Pleistoceno de la Provincia de Córdoba. Ameghiniana, 36 : 55-62.

Ubilla, M.; Perea, D.; Aguilar, C.G. \& Lorenzo, N. (2004). Late Pleistocene vertebrates from northern Uruguay: tools for biostratigraphic, climatic and environmental reconstruction. Quaternary International, 114: 129-142. doi:10.1016/S1040-6182(03)00048-X

Villa, P. \& Mahieu, E. (1991). Breakage patterns of human long bones. Journal of Human Evolution, 21: 27-48. doi:10.1016/0047-2484(91)90034-S

Von den Driesch, A. (1976): A Guide to the Measurement of Animal Bones from Archaeological Sites. Peabody Museum Bulletin, 1, Cambridge Mass, 136.

Voorhies, M. (1969). Taphonomy and Population Dynamics of an Early Pliocene Vertebrate Fauna, Knox County, Nebraska. Contributions to Geology Special Paper, no. 1. University of Wyoming, Laramie, $69 \mathrm{pp}$.

Weeb, S.D. (1991). Ecogeography and the Great American Interchange. Paleobiology, 17: 266-280.

Webb, S.D. \& Stehli, F.G. (1995). Selenodont Artiodactyla (Camelidae and Cervidae) from the Leisey Shell Pits, Hills Borough County, Florida. Bulletin of the Florida Museum of Natural History, 37: 621-643.

Weinstock, J.; Shapiro, B.; Prieto, A.; Marín, J.C. González, B.A.; Gilbert, P.; Thomas, M. \& Willerslev, E. (2009). The late Pleistocene distribution of vicuñas ( Vicugna vicugna) and the "extinction" of the gracile llama ("Lama gracilis"): New molecular data. Quaternary Science Reviews, 28: 1369-1373. doi:10.1016/ j.quascirev.2009.03.008

Woodburne, M.O.; Cione, A.L. \& Tonni, E.P. (2006). Central American provincialism and the Great American Biotic Interchange. In: Advances in late Tertiary vertebrate paleontology in Mexico and the Great American Biotic Interchange (Carranza-Castañeda, Ó. \& Lindsay, E. H., eds.). Universidad Nacional Autónoma de México, Instituto de Geología y Centro de Geociencias, Publicación Especial 4, 73-101.

Recibido el 24 de febrero de 2012 Aceptado el 19 de julio de 2012 Publicado online el 15 de abril de 2013 